\title{
Collective Decision-Making on Triadic Graphs
}

\author{
Ilja Rausch, Yara Khaluf, and Pieter Simoens \\ IDLab - Department of Information Technology \\ Ghent University - imec, Technologiepark 126, B-9052 Ghent, Belgium \\ ilja.rausch@ugent.be
}

\begin{abstract}
Many real-world networks exhibit community structures and non-trivial clustering associated with the occurrence of a considerable number of triangular subgraphs known as triadic motifs. Triads are a set of distinct triangles that do not share an edge with any other triangle in the network. Network motifs are subgraphs that occur significantly more often compared to random topologies. Two prominent examples, the feedforward loop and the feedback loop, occur in various real-world networks such as gene-regulatory networks, food webs or neuronal networks. However, as triangular connections are also prevalent in communication topologies of complex collective systems, it is worthwhile investigating the influence of triadic motifs on the collective decision-making dynamics. To this end, we generate networks called Triadic Graphs (TGs) exclusively from distinct triadic motifs. We then apply TGs as underlying topologies of systems with collective dynamics inspired from locust marching bands. We demonstrate that the motif type constituting the networks can have a paramount influence on group decision-making that cannot be explained solely in terms of the degree distribution. We find that, in contrast to the feedback loop, when the feedforward loop is the dominant subgraph, the resulting network is hierarchical and inhibits coherent behavior.
\end{abstract}

Keywords: complex networks, triadic motifs, collective decision-making, group coherence, feedforward loop, hierarchality

\section{Introduction}

The network topology defining agent interactions plays a crucial role in swarminspired collective systems $[4,8,11,14,17]$. Researchers are beginning to unravel the influence of the topology on collective decision-making and propose engineering approaches that increase the performance of (artificial) collective systems $[11,14,16]$. To gain insights into how interaction networks impact decisionmaking, a popular approach is to generate well defined topologies that are inspired from real-world observations. Often these topologies are designed to reduce complexity and focus on the impact of specific network properties.

One of the properties worth investigating is the presence of triangular connections. Recently, an abundance of triangular subgraphs called triadic motifs has been discovered in numerous real-world networks $[13,15,21]$. Particularly, the 
feedforward loop was found, among others, in gene-regulatory networks, food webs or neuronal networks $[1,15,21]$.

In light of these discoveries, a reliable method was developed for generating graphs with an abundance of such motifs, called Triadic Random Graphs (TRGs). A TRG is generated using exclusively triadic motifs $\mathcal{M}_{i}$ as building blocks [20]. This approach is based on conditionally independent sampling of triples from Steiner Triple Systems and the assignment of the motif-specific topology to each triple. As a result, the homogeneous TRG $\mathcal{T}_{i}$ is a directed graph that consists purely of $T$ distinct triadic subgraphs of type $\mathcal{M}_{i}$. The benefits of TRGs are twofold: (i) the dynamics emerging on the local level of each triadic motif are directly influenced through no more than three nodes, i.e. higher-order correlations are reduced, (ii) the global network topology can be purely described in terms of motif type $\mathcal{M}_{i}$ and $T$ (no higher order motif analysis is required). Network properties such as average degree, clustering coefficient or shortest path can be controlled, at least to some extent, by the choice of $\mathcal{M}_{i}$ and $T$. Thus, TRGs can provide a simple but powerful fundamental design for studying the impact of triadic connections on complex dynamic processes.

An increasingly popular example of complex dynamics is the collective decisionmaking observed in locust marching bands $[2-4,8,16]$. In essence, it represents a symmetry-breaking scenario in which a large group faces the choice between two equal-quality options. In dense groups, the corresponding communication network displays a high number of triangular connections whose impact on the group behavior is not yet fully understood $[8,11]$. In this paper we aim at enhancing this understanding by studying the influence of triadic motifs on the locust-inspired symmetry-breaking model.

A common approach for modeling swarming systems such as locust marching bands, is to allow the individuals influence each other's behavior based on physical proximity (i.e. euclidean distance) $[2,6,10,18]$. However, to focus the analysis solely on the contribution of the motif topology, the approach taken in the current study is different in that we only consider the non-euclidean, static group communication network, disregarding correlations in physical space. This approach is necessary as it allows applying pre-constructed well-defined triadic graphs and, thus, comparing the contribution of the different motif types in a controlled way. The findings obtained this way can then serve as a baseline for more sophisticated methods such as adaptive-network models $[4,8,9]$.

Nevertheless, as we will show below, our network-driven approach is sufficient to reproduce the main characteristics of state trajectories known from the swarming model $[2,3]$. Moreover, the network approach offers the possibility to confirm the validity of the previously analytically derived upper limit to the coherence degree $\overline{|\phi|}$ (a measure of the group alignment) [16]. In particular, we will demonstrate that for some motif types, the group may reach this upper bound of $\overline{|\phi|}$ while other motifs have a comparably inhibitory impact. As we will show, a shared characteristic of the latter motif types is the presence of nodes with zero out-degree. Decreasing their number resulted in an increase of $\overline{|\phi|}$. Finally, we observed that the use of the feedforward loop led to a hierarchical network 
structure that was particularly detrimental to the group alignment. Therefore, our results suggest that the motif type can have important consequences for collective decision-making.

We introduce the TRGs in Sec. 2 and the locust-inspired collective decisionmaking model in Sec. 3. Subsequently, we report and discuss our results in Sec. 4, and conclude in Sec. 5 .

\section{Triadic Graphs}

\subsection{Triadic Motifs}

In the current study we focus on the directed closed triadic motifs $\mathcal{M}_{i}$ illustrated in Fig. 1. Among these, are the feedforward loop $\mathcal{M}_{1}$, the feedback loop $\mathcal{M}_{3}$ and the bi-directional loop $\mathcal{M}_{7}$. To investigate the influence of triadic motifs on the collective decision-making we generated seven types of TGs, $\mathcal{T}_{i}$, following a procedure described below. For each $\mathcal{T}_{i}$ only one type of triadic motifs, $\mathcal{M}_{i}$, was used as building block, respectively. In each $\mathcal{T}_{i}$ every node is an element of at least one triadic motif. The total number of distinct triadic motifs was verified using the MFINDER software (version 1.2) ${ }^{1}$.

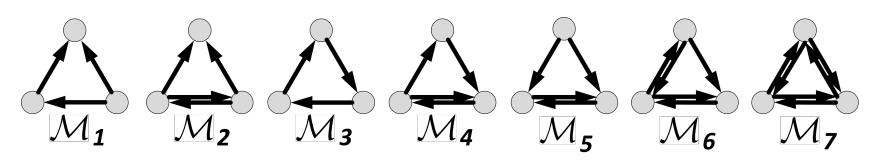

Fig. 1. The seven types of triadic motifs used as building blocks of TGs $\mathcal{T}_{i}$.

\subsection{Steiner Triple Systems}

A recent mechanism proposed by [20] specializes in network construction using triadic motifs as primary building blocks. This mechanism is based on the concept of Steiner Triple Systems (STS), a mathematical design of a structure consisting of distinct three element subsets (i.e. triples). The most profound feature of the STS is that any pair of elements can be connected through only one unique link that belongs to exactly one triad. Therefore, a network based on Steiner Triples contains exclusively distinct triadic subgraphs. To realize an STS with $N$ elements, two necessary and sufficient conditions need to be satisfied [12]:

$$
N \bmod 2=1, N(N-1) \bmod 3=0
$$

leading to an upper bound for the number of distinct triads $T$ [20]:

$$
T \leq \frac{1}{3} \frac{N(N-1)}{2} .
$$

\footnotetext{
${ }^{1}$ https://www.weizmann.ac.il/mcb/urialon/
} 
To satisfy Eq. (1)-2, all TRGs generated in the current study have a size of $N=7^{3}=343$ and $T=343 n$ with $n \in \mathbb{N}<8$.

\subsection{Triadic Graphs}

Similar to the Erdős-Rényi model, where the assignment of edges is not conditioned on the current state of the network, in the model proposed by [20] the triad assignment is conditionally independent and the resulting graph is the TRG. A key aspect of this approach is that $T$ triads are sampled from a pre-constructed STS $G_{S T S}$. Each sampled triad is assigned the motif-specific topology (such as those shown in Fig. 1). Therefore, triadic motifs are the fundamental parts constituting (directed) TRGs. Note that this model allows the generation of random network ensembles that have the same number of edges and nodes.

However, to ensure that every generated network has only one connected component, we slightly deviated from the above-mentioned procedure. For each instance, before independently assigning the triads, we first created a seed network $G_{s}$ (an example is shown in Fig. 2 (left)) by iterating through all nodes of $G_{S T S}$ and assigning a triad $\theta \in G_{S T S}$ to $G_{S}$ only if $\theta$ satisfies the following two conditions: (i) at least one node $(\nu \in \theta) \wedge\left(\nu \notin G_{s}\right)$, (ii) at least one $(\nu \in \theta) \wedge\left(\nu \in G_{s}\right)$. As a result, $G_{s}$ has only one connected component with the same predefined number of nodes $N$ as the $G_{S T S}$ from which the triads are sampled. Subsequently, to reach the predefined number $T, G_{s}$ is assigned new triads by means of randomly drawing new $\theta$ from $G_{S T S}$ without replacement. Fig. 2 (right) shows an example of a final TRG $\mathcal{T}_{1}$. Due to this procedure the graphs are not as strictly random as the original [20], thus we will henceforth refer to them as Triadic Graphs (TGs).
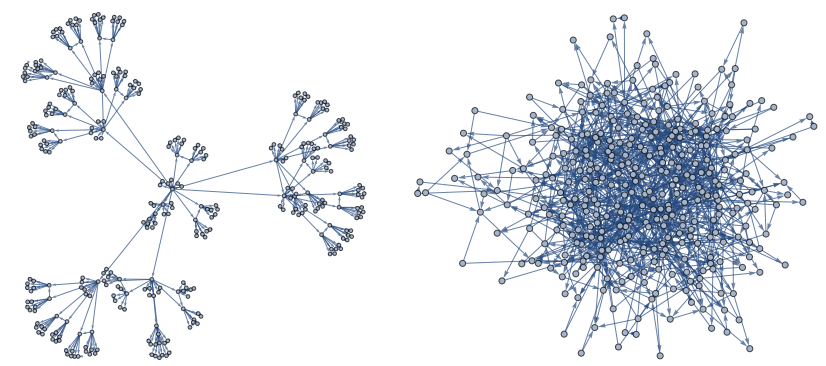

Fig. 2. Examples of Left: a seed network $G_{s}$, Right: a final TG. Both graphs were constructed from motifs of type $\mathcal{M}_{1}$.

We distinguish between homogeneous and heterogeneous TGs. In the former case the total number of triads is $T=T_{i}$, with $T_{i}$ being the number of motifs of type $\mathcal{M}_{i}$. Whereas, in a heterogeneous TG, $T=\sum_{i=1}^{7} T_{i}$. The choice of the particular type of $\mathcal{M}_{i}$ for TG construction strongly determines various network properties, particularly the degree distribution. 
The total degree of a node $\nu$ is given by $k_{t o t, \nu}=k_{i n, \nu}+k_{o u t, \nu}$, where $k_{i n, \nu}$ and $k_{\text {out }, \nu}$ are the node's in- and out-degree, respectively. For instance, consider the right-most node of $\mathcal{M}_{1}$ in Fig. 1 . Its degree is given by $k_{\text {in }, \nu}=0, k_{\text {out }, \nu}=2$ and $k_{t o t, \nu}=2$. In contrast, the degree of the analogous node of $\mathcal{M}_{2}$ is given by $k_{i n, \nu}=1, k_{\text {out }, \nu}=2$ and $k_{t o t, \nu}=3$. As the triadic motifs are assigned conditionally independent of each other, the number of triadic motifs around $\nu$ is binomially distributed for small TGs and Poisson distributed for large TGs. Moreover, the global average of the total degree is correlated with the type of $\mathcal{T}_{i}$. For instance, consider again the motifs $\mathcal{M}_{1}$ and $\mathcal{M}_{7}$. The total in-degree, summed over all three nodes, is $k_{i n, \mathcal{M}_{1}}=3$ and $k_{i n, \mathcal{M}_{7}}=6$, respectively (and similarly for the out-degree). Thus, the total degree distribution of a network is a function of the type and number of triadic motifs and can be deduced from the TG model [20]. Consequently, the average total degree can be calculated as a function of $\mathcal{T}_{i}:\left\langle k_{\text {tot }}\right\rangle=\frac{T_{i}}{N}\left(k_{\text {in }, \mathcal{M}_{i}}+k_{\text {out }, \mathcal{M}_{i}}\right)$.

Finally, it can be useful to isolate the influence of the degree distribution on the dynamical processes from that of the triadic motifs. For this purpose, we compare the simulation outcomes of TGs to null-models which are the respective randomizations. The randomized version of each TG is generated using the a Markov Chain Monte Carlo rewiring algorithm [19]. In essence, the algorithm runs a predefined number of mutually independent, degree-preserving rewirings between pairs of nodes. As a consequence, the initial abundance of triadic motifs vanishes while the network size, the number of connections and the distribution of in- and out-degrees are preserved.

\section{Decision making model}

We examine the influence of triadic motifs on the decentralised decision-making dynamics in the canonical model of locust marching $[3,8,11,16]$. This biologically inspired model represents a binary decision problem in which the agents need to collectively decide whether to go left or right $[2,6,22]$.

Consider a one-dimensional opinion space $o_{i}$ of an individual $i$, in which a commitment to option $A(B)$ corresponds to $o_{i}<0\left(o_{i}>0\right)$, respectively. Then, similar to the locust velocity $[2,16,22]$, the opinion of $i$ is updated at each time step to

$$
o_{i}(t+1)=\delta_{s}\left[G\left(\left\langle o_{i}(t)\right\rangle\right)+\zeta_{i}(t)\right],
$$

where $\zeta_{i}(t) \in[-1.0,1.0]$ is a real random number sampled from a uniform distribution (representing noise). Additionally, the average opinion of the individual's neighborhood $\left\langle o_{i}(t)\right\rangle$ is given by $\left\langle o_{i}(t)\right\rangle=\frac{1}{k_{i n, i}} \sum_{j=0}^{N} o_{j}(t) A_{j i}$, where $A_{j i}$ is an element of the network's $N \times N$ adjacency matrix, with $A_{j i}=1$ if there is a direct link leading from $j$ to $i$ and $A_{j i}=0$ otherwise. As a common simplification, self-loops are excluded, i.e. $A_{i i}=0$ for all $i ; k_{i n, i}=\sum_{j=0}^{N} A_{j i}$ is the $i$ 's total in-degree. Moreover, the contribution of the individual's neighbors is maintained close to \pm 1.0 by the piece-wise continuous function

$$
G\left(\left\langle o_{i}(t)\right\rangle\right)=\frac{1}{2}\left[\left\langle o_{i}(t)\right\rangle+\operatorname{sgn}\left(\left\langle o_{i}(t)\right\rangle\right)\right],
$$


with $\operatorname{sgn}()$ being the sign-function. Note that for $G\left(\left\langle o_{i}(t)\right\rangle\right)=\left\langle o_{i}(t)\right\rangle$, Eq. (3) would be similar to the majority vote model. Finally, $\delta_{s}$ in Eq. (3) is a binary digit that is $\delta_{s}=-1$ with probability $p_{s}$ and $\delta_{s}=1$ otherwise. While the Czirók model is obtained for $p_{s}=0$, setting $p_{s}>0$ extends this model and represents the ability of each individual to spontaneously change its opinion $[4,8,11]$. This spontaneous opinion switch does not necessarily represent noise but can also be attributed to the individual's drive to explore alternatives and perpetuate selforganization. Alternatively, it could be a malicious behavior originating from external attacks or a similar underlying mechanism unknown to the observer [16].

Similar to $[11,16]$, the collective opinion of the $N$ individuals, i.e. the collective state of the system is given by:

$$
\phi(t)=\frac{1}{N} \sum_{i=0}^{N} \operatorname{sgn}\left(o_{i}(t)\right) .
$$

The collective coherence degree $|\phi(t)|$ is defined as the absolute value of the collective opinion. When all individuals agree on an option the system reaches consensus with $|\phi(t)|=1$.

Beside the spontaneous switching, there are two further significant differences between the standard locust marching model and the decision-making model considered here. First, unlike the previous studies, we do not include the spatial information of the individuals. Instead, we only focus on the opinion dynamics from an abstract network-driven perspective. While previously the neighbors were selected from the spatial proximity of an individual, i.e. within a certain range $\Delta$ around the individual $[2,3,6,16,22]$, or from an adaptive network $[4,8]$, here the neighbors are assigned by the adjacency matrix of a predefined static network. Thus, we intentionally isolate the problem from spatial correlation and dynamic link rewiring to focus exclusively on the impact of the subtle differences between the triadic motifs. Second, due to to the directed nature of the triadic motifs, the considered networks are directed which is in contrast to most previous works that focused mainly on bidirectional communication.

\section{Results and Discussion}

First, we demonstrate that Eq. (3)-Eq. (5) can qualitatively reproduce the main characteristics of the empirically observed locust alignment trajectories $[2,6,22]$. For this, we simulated the collective decision making on TGs in various configurations. The initial number of individuals with $o_{i}>0$ and $o_{i}<0$ was always $\left\lceil\frac{N}{2}\right\rceil$ and $N-\left\lceil\frac{N}{2}\right\rceil$, respectively. The example shown in Fig. 3 is the simulation outcome for three instances of $\mathcal{T}_{1}$ - which differ only in $T$ - that illustrates the qualitative similarities to three major empirical findings: (i) for high enough communication degree, $\phi(t)$ fluctuates around a non-trivial stable state with a residence time $\tau \geq \tau_{\text {tot }}$ (dark blue data with $\left\langle k_{t o t}\right\rangle=24$ ); the residence time $\tau$ represents the time interval between two state transitions while $\tau_{\text {tot }}$ is the total experiment duration, with $\tau_{\text {tot }}=5000 t s$ for all our experiments; (ii) collective state may repeatedly undergo rapid transitions between temporary stable 
states with $1 \ll \tau<\tau_{\text {tot }}$ (blue data with $\left\langle k_{t o t}\right\rangle=12$ ); (iii) lower communication degree leads to lower group alignment and $\tau \rightarrow 1$ (light blue data with $\left.\left\langle k_{t o t}\right\rangle=6\right)[11,16]$.
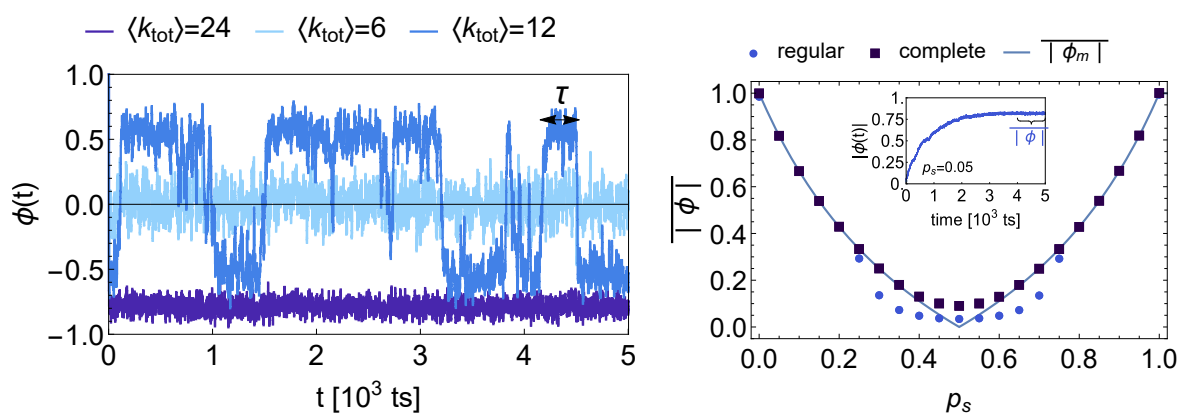

Fig. 3. Left: Collective state trajectories for $p_{s}=0.05$ and three $\mathcal{T}_{1}$ instances with different $T$ leading to different values of $\left\langle k_{t o t}\right\rangle$. Each trajectory represents one sample, i.e. here the simulations were run with the same random number generator seed. An example of the residence time $\tau$ is illustrated for one of the temporary states. Right: Coherence degree $\overline{|\phi(t)|}=\overline{|\phi|}$ over $p_{s}$ for a complete and a regular network with $\left\langle k_{t o t}\right\rangle=48$, both networks with $N=343$. The inset shows the period over which the time average $\overline{|\phi|}$ of the collective state was taken.

Moreover, the maximum value of $\overline{|\phi(t)|}$ obtained from simulation agrees well with previous theoretical findings [16] suggesting an upper bound of:

$$
\left|\phi_{m}\right|=\frac{\left|0.5-p_{s}\right|}{1-\left|0.5-p_{s}\right|} .
$$

Fig. 3 (right) showcases this agreement for complete and regular networks. Each $\overline{|\phi|}$ trajectory was averaged over 50 simulation runs. As illustrated in the inset of Fig. 3 (right), each data point represents the time average of $\overline{|\phi|}$ obtained over the last 1000 time steps to account for a transient period occurring in the early stages of the simulations. Similar to [16], the global coherence degree does not exceed the limit given by Eq. (6) for the regular network of $\left\langle k_{t o t}\right\rangle=48$ (this $\left\langle k_{t o t}\right\rangle$ value is comparable to the high-degree simulations in [16]). Additionally, as expected for the complete network, $\overline{|\phi|}$ exceeds $\overline{\left|\phi_{m}\right|}$ only for $p_{s} \approx 0.5$, i.e. where Eq. (6) is not well defined. Note that for $p_{s}>0.5, \overline{|\phi|}$ increases. For these $p_{s}$ the agent is likely to switch its opinion within the same time step as its neighbours, leading to exceedingly synchronous switching and higher $\overline{|\phi|}[16]$.

To investigate the influence of the motifs, it is worthwhile examining the collective ability to achieve $\overline{\left|\phi_{m}\right|}$ for different $\mathcal{T}_{i}$. Fig. 4 (left) shows the simulation outcome for different values of the spontaneous switch probability $p_{s}$. As Fig. 4 suggests, $\overline{|\phi|}$ can be influenced by the choice of $\mathcal{M}_{i}$ although the results are comparably similar for most motifs. The differences between the TGs with motifs 
$\mathcal{M}_{1-2}$ and $\mathcal{M}_{3-7}$ are particularly apparent with $\mathcal{M}_{1}$ and $\mathcal{M}_{2}$ having the largest inhibitory impact. This is reflected in the deviation of $\overline{|\phi|}$ from $\overline{\left|\phi_{m}\right|}$. On the one hand, for some values of $p_{s}$, this deviation more than doubles between the TGs $\mathcal{T}_{1}$ and $\mathcal{T}_{3-7}$ (see inset of Fig. 4 (left)). On the other hand, the coherence degree results are remarkably similar from $\mathcal{T}_{3}$ to $\mathcal{T}_{7}$.
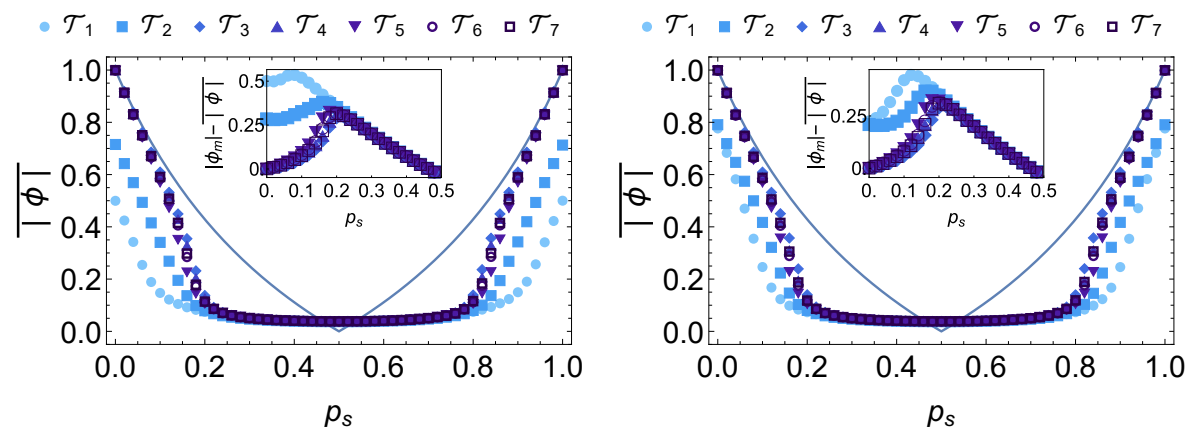

Fig. 4. Coherence degree as a function of $p_{s} \in[0,1]$ and the network topology. For all networks, $N=343$ and $\left\langle k_{t o t}\right\rangle=24$; The curve represents the theoretical value of maximum coherence degree $\left|\phi_{m}\right|$. Left: TGs $\mathcal{T}_{i}$, Right: Null-models of $\mathcal{T}_{i}$. The insets show the respective differences between the theoretical value $\left|\phi_{m}\right|$ and the measured time averaged coherence degree $\overline{|\phi|}$.

To obtain a more detailed view on the impact of the motif topology, we generated null-models (i.e. degree-preserving TG randomizations as described in Sec. 2.3). The decision-making results for the null-models are shown in Fig. 4 (right). Strikingly, the simulation outcomes for $\mathcal{T}_{3-7}$ are very close to their randomized counterparts and the only noticeable difference is observed for $\mathcal{T}_{1}$, i.e. the TG generated from feedforward loops $\mathcal{M}_{1}$. This observation indicates that the $k_{\text {in }}$ and $k_{\text {out }}$ distributions have a more critical impact on $\overline{|\phi|}$ than $\mathcal{M}_{i}$, with the exception of $\mathcal{M}_{1}$. In Fig. 4 (right) one can see that despite the absence of motifs, the null-models of $\mathcal{T}_{1}$ and $\mathcal{T}_{2}$ still have an inhibitory impact on $\overline{|\phi|}$ in comparison to the other graphs. A shared characteristic of $\mathcal{T}_{1}$ and $\mathcal{T}_{2}$ is the presence of nodes with $k_{\text {out }}=0$ that are unable to communicate their opinion. Interestingly, the inhibitory effects of nodes with $k_{i n}=0$ are lower as evidenced by the comparison with $\mathcal{T}_{5}$ where all nodes have $k_{\text {out }}>0$ but some nodes may have $k_{i n}=0$. Nodes with $k_{i n}=0$ are not affected by their neighborhood and are thus similar to 'stubborn' individuals commonly referred to as zealots [5].

Moreover, as was shown previously, the coherence degree is strongly correlated with $\left\langle k_{t o t}\right\rangle[16]$. Higher $\left\langle k_{t o t}\right\rangle$ leads to higher $\overline{|\phi|}$, up to $\overline{\left|\phi_{m}\right|}$. This behaviour can also be observed for TGs by increasing $T$, as shown in Fig. 5 for $\mathcal{T}_{1}$ (left) and $\mathcal{T}_{3}$ (right). One can see that for a range of $p_{s}$ values even after increasing $\left\langle k_{t o t}\right\rangle$ by a factor of eight (i.e. for $T=343 \times 8=2744$ ), $\mathcal{T}_{1}$ is associated with lower $\overline{|\phi|}$ than $\mathcal{T}_{3}$. 

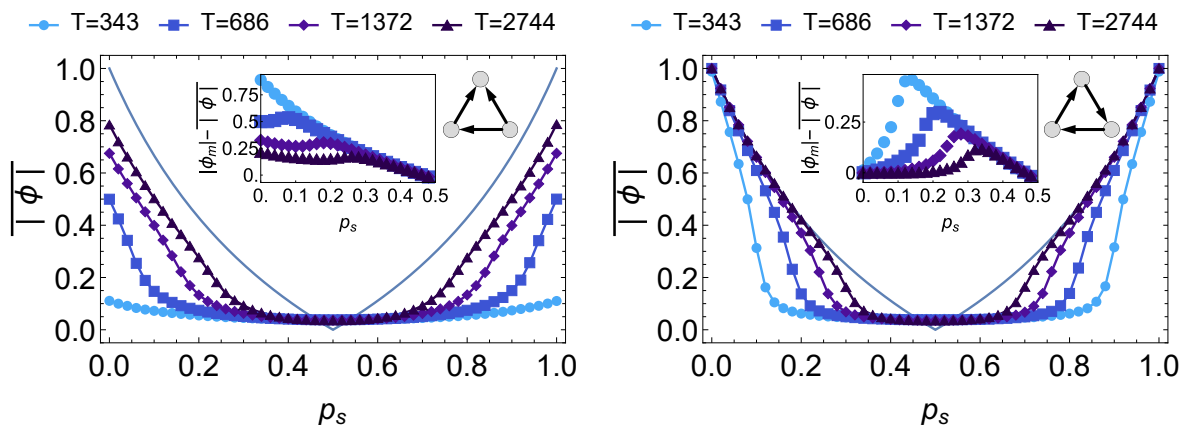

Fig. 5. Left: Simulation outcome for $\mathcal{T}_{1}$. Right: Simulation outcome for $\mathcal{T}_{3}$. In both plots, the lines between the data points are guides to the eye. The $\mathrm{V}$-shaped curve indicates the theoretical $\overline{\left|\phi_{m}\right|}$. In each panel the insets show the corresponding difference $\overline{\left|\phi_{m}\right|}-\overline{|\phi|}$ as well as an illustration of the building block motif $\mathcal{M}_{i}$.

Transitioning from $\mathcal{T}_{1}$ to $\mathcal{T}_{3}$ by means of gradually replacing quantities of $\mathcal{M}_{1}$ with $\mathcal{M}_{3}$ leads to a decrease of nodes with $k_{\text {in }}=0$ and $k_{\text {out }}=0$ together with an increase of $\overline{|\phi|}$. This observation is demonstrated in Fig. 6 (left) where the data was collected for a set of heterogeneous TGs with $T_{3}$ motifs of type $\mathcal{M}_{3}$ and $T_{1}=T-T_{3}$ motifs of type $\mathcal{M}_{1}$ (and for $p_{s}=0.05$ ). Each heterogeneous TG had on average $k_{\text {out }}^{0}$ nodes with $k_{\text {out }}=0$ that decreased linearly with $T_{3}$ and reached zero for $T_{3} \approx 231$ (with $\overline{|\phi|} \approx 0.64$ ). In contrast, the coherence degree increased with $T_{3}$ non-linearly. Thus, the inhibitory impact of $\mathcal{M}_{1}$ and $\mathcal{M}_{2}$ can only partially be explained by the presence of nodes with $k_{\text {out }}=0$.
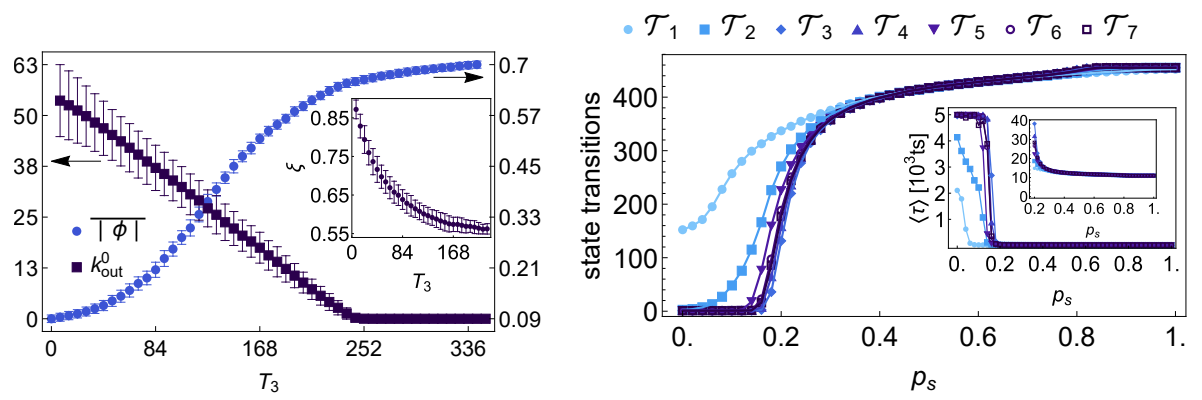

Fig. 6. Left: Number of nodes with $k_{\text {out }}=0$ and $\overline{|\phi|}$ for the transition from $\mathcal{T}_{1}$ to $\mathcal{T}_{3}$ by replacing $T_{3}$ motifs of type $\mathcal{M}_{1}$ with $\mathcal{M}_{3}$ for $p_{s}=0.05$. Inset: Current parameter $\xi$ as a function of $T_{3}$. Right: Number of state transitions within 5000 time steps of simulation. The inset shows the average residence time $\langle\tau\rangle$ and a related close-up view for $p_{s} \geq 0.2$. The lines are guides to the eye. 
Another relevant feature that changes with the transition from $\mathcal{T}_{1}$ to $\mathcal{T}_{3}$ is the current parameter $\xi$. It is a measure for the hierarchality or the inherent directionality of the system [7]. In the presence of nodes with $k_{\text {out }}=0$ it can be easily obtained and it essentially represents the fraction of links that point upwards the hierarchical node ordering. Consequently, $\xi=1$ in a perfectly hierarchical structure with very strong inherent directionality and $\xi \approx 0.5$ in a random graph with a vanishing level of inherent directionality and hierarchy [7]. For the set of homogeneous TGs $\mathcal{T}_{i}$ (as those used in Fig. 4), our measurements yielded $\xi=$ $\{0.85 \pm 0.03,0.50 \pm 0.01,0.54 \pm 0.01,0.51 \pm 0.01,0.63 \pm 0.07,0.50 \pm 0.01,0.50 \pm 0.00\}$, where the first element, $\xi_{1}$, corresponds to $\mathcal{T}_{1}$, the second, $\xi_{2}$, to $\mathcal{T}_{2}$, etc.. Note that while $\xi_{1} \approx 0.85$, indicating strong hierarchality, $\xi_{2} \approx 0.5$ indicating that the hierarchical property of $\mathcal{T}_{2}$ is almost non-existent. This suggests that the inhibitory impact of $\mathcal{M}_{2}$ on group coherence is mainly due to the presence of nodes with $k_{\text {out }}=0$ (of which there are $k_{\text {out }}^{0}=34 \pm 11$ ). This is in line with the observations that in contrast to $\mathcal{T}_{1}$, degree-preserved randomization of $\mathcal{T}_{2}$ does not significantly improve $\overline{|\phi|}$ (see Fig. 4).

Finally, Fig. 6 (right) shows that $\mathcal{M}_{1}$ and $\mathcal{M}_{2}$ increase the number of state transitions and, consequently, decrease the average residence time $\langle\tau\rangle$. The differences between the $\mathcal{T}_{i}$ rapidly vanish for $p_{s}>0.2$ (see inset of Fig. 6 (right)). However, for $p_{s} \leq 0.2$ the higher number of state transitions of $\mathcal{T}_{1}$ suggest a potentially higher level of group adaptivity. Changing the state enables the group to explore the properties of this state and reassess its quality. Conversely, maintaining a stable state enables exploitative behavior. Motifs $\mathcal{M}_{3}-\mathcal{M}_{7}$ appear to be beneficial for the latter while $\mathcal{M}_{1}$ and $\mathcal{M}_{2}$ for the former behavior.

\section{Conclusion}

In a binary collective decision-making task, noise coupled with social feedback mechanisms allow an initially symmetric system to self-organize and converge towards one of the two options. Social feedback mechanisms are defined by the interactions between the individuals, i.e. their underlying communication network. Therefore, it is paramount to understand the role of the network topology in promoting or inhibiting collective decision-making and coordination.

To investigate the impact of particular network types that include an abundance of triadic motifs, we generated random graphs that consist exclusively from such motifs. These graphs were then applied in simulations of binary collective decision-making scenarios. The results have shown that two specific types of motifs, in particular the feedforward loop, have a strong inhibitory impact on the coherence of collective behavior $\overline{|\phi|}$. In contrast, with motifs such as the feedback loop or the bidirectional loop (the motif in which all links are bidirectional), the system was able approach maximum coherence. Moreover, through comparison to null-models we have shown that the latter motif types achieve similar $\overline{|\phi|}$ to their degree-preserved randomizations, indicating that the in- and out-degree distributions may be the more critically influential properties. 
More importantly, these results demonstrate that a number of motifs influence collective decision-making similarly to the bi-directional motif that can also be interpreted as undirected. On the one hand, this suggests that, depending on the purpose of the study, increasing the system complexity by including directionality may not be necessary. On the other hand, it appears that a group can reach maximum coherence even when local relationships are considerably asymmetric (compare the feedback loop to the bi-directional loop). This observation essentially lifts the constraint of symmetric relationships in which both nodes along an edge need to communicate to each other.

However, not all motifs lead to similar results and, particularly, the feedforward loop stands out with having a comparably inhibiting impact on the group coherence. We identified two possible characteristics, resulting from the motif topology, that contribute to such influence on collective decision-making. First, it is the presence of nodes with zero out-degree, i.e. nodes that do not communicate their opinion to their neighborhood. Surprisingly, their counterparts, nodes with zero in-degree, appear to not have similar inhibitory impact on collective decision-making. In fact, the motif where one node has zero in-degree but all three nodes have above-zero out-degree leads to similar group coherence as the feedback loop or the bi-directional loop but not as the feedforward loop. Second, the abundance of feedforward loops leads to hierarchical structures that are not beneficial to opinion alignment in group decision-making. Moreover, it leads to comparably unstable group commitment and a higher number of transitions between the options. Therefore, our results suggest that in certain cases the motif topology can have important consequences on collective decision-making.

In future research, it is worthwhile investigating in more detail the precise reasons behind the inhibitory influence of the prominent feedforward loop on collective decision-making, particularly with focus on the role of nodes with zero out-degree and hierarchality. Other network properties such as the path length or centrality may be relevant and should be included in the analysis. Moreover, the ability to lift the constraints of symmetrical relationships allows to examine scenarios of heterogeneous societies in which nodes are assigned particular roles based on the motif topology. Finally, the study can be further extended to include stochastic models as well as other motif types such as open triadic or quadratic motifs.

\section{References}

1. Alon, U.: Network motifs: theory and experimental approaches. Nat. Rev. Genet. 8(6), 450-461 (2007)

2. Ariel, G., Ayali, A.: Locust collective motion and its modeling. PLoS Comput. Biol. 11(12), e1004522 (2015)

3. Buhl, J., Sumpter, D.J., Couzin, I.D., Hale, J.J., Despland, E., Miller, E.R., Simpson, S.J.: From disorder to order in marching locusts. Science 312(5778), 1402-1406 (2006)

4. Chen, L., Huepe, C., Gross, T.: Adaptive network models of collective decision making in swarming systems. Phys. Rev. E 94(2), 022415 (2016) 
5. Colaiori, F., Castellano, C.: Consensus versus persistence of disagreement in opinion formation: the role of zealots. J Stat Mech: Theory E 2016(3), 033401 (2016)

6. Czirók, A., Barabási, A.L., Vicsek, T.: Collective motion of self-propelled particles: Kinetic phase transition in one dimension. Phys. Rev. Lett. 82, 209-212 (1999)

7. Domínguez-García, V., Pigolotti, S., Muñoz, M.A.: Inherent directionality explains the lack of feedback loops in empirical networks. Sci. Rep. 4, 7497 (2014)

8. Huepe, C., Zschaler, G., Do, A.L., Gross, T.: Adaptive-network models of swarm dynamics. New J. Phys. 13(7), 073022 (2011)

9. Khaluf, Y., Hamann, H.: Modulating interaction times in an artificial society of robots. In: The 2018 Conference on Artificial Life (ALIFE). pp. 372-379. MIT Press (2019)

10. Khaluf, Y., Pinciroli, C., Valentini, G., Hamann, H.: The impact of agent density on scalability in collective systems: Noise-induced versus majority-based bistability. Swarm Intelligence 11(2), 155-179 (2017)

11. Khaluf, Y., Rausch, I., Simoens, P.: The impact of interaction models on the coherence of collective decision-making: A case study with simulated locusts. In: Dorigo, M., Birattari, M., Blum, C., Christensen, A.L., Reina, A., Trianni, V. (eds.) Swarm Intelligence: 11th International conference, ANTS 2018, volume 11172 of LNCS, pp. 252-263. Springer, Cham (2018)

12. Kirkman, T.P.: On a problem in combinations. Cambridge and Dublin Math. J 2(191-204), 1847 (1847)

13. Klaise, J., Johnson, S.: The origin of motif families in food webs. Sci. Rep. 7(1), 16197 (2017)

14. Mateo, D., Horsevad, N., Hassani, V., Chamanbaz, M., Bouffanais, R.: Optimal network topology for responsive collective behavior. Sci. Adv. 5(4) (2019)

15. Milo, R., Shen-Orr, S., Itzkovitz, S., Kashtan, N., Chklovskii, D., Alon, U.: Network motifs: Simple building blocks of complex networks. Science 298(5594), 824-827 (2002)

16. Rausch, I., Reina, A., Simoens, P., Khaluf, Y.: Coherent collective behaviour emerging from decentralised balancing of social feedback and noise. Swarm Intell. 13(3), 321-345 (2019)

17. Shang, Y., Bouffanais, R.: Influence of the number of topologically interacting neighbors on swarm dynamics. Sci. Rep. 4, 4184 (2014)

18. Vicsek, T., Czirók, A., Ben-Jacob, E., Cohen, I., Shochet, O.: Novel type of phase transition in a system of self-driven particles. Phys. Rev. Lett. 75, 1226-1229 (Aug 1995)

19. Winkler, M., Reichardt, J.: Node-specific triad pattern mining for complex-network analysis. In: 2014 IEEE International Conference on Data Mining Workshop. pp. 605-612. IEEE Press, New York (2014)

20. Winkler, M., Reichardt, J.: Motifs in triadic random graphs based on steiner triple systems. Phys. Rev. E 88, 022805 (Aug 2013)

21. Yao, Y., Carretero-Paulet, L., Van de Peer, Y.: Using digital organisms to study the evolutionary consequences of whole genome duplication and polyploidy. PLOS ONE 14(7), 1-21 (07 2019)

22. Yates, C.A., Erban, R., Escudero, C., Couzin, I.D., Buhl, J., Kevrekidis, I.G., Maini, P.K., Sumpter, D.J.T.: Inherent noise can facilitate coherence in collective swarm motion. P. Natl. Acad. Sci. 106(14), 5464-5469 (2009) 
We use cookies to personali

features and to analyse our traffic. We also share information about your use of our site with our social media, advertising and analytics partners in accordance with our Privacy Policy. You can manage your preferences in Cookie Settings.

» Physics » Complexity

Springer Proceedings in Complexity

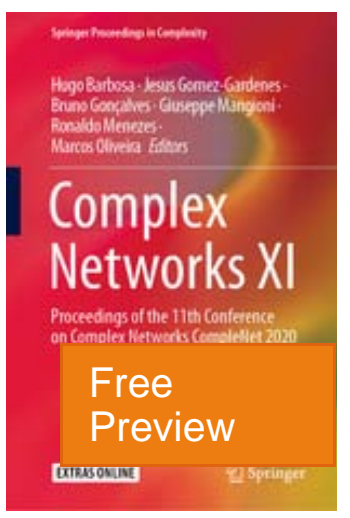

(C) 2020

\section{Complex Networks XI}

Proceedings of the 11th Conference on Complex Networks CompleNet 2020

Editors: Barbosa, H., Gomez-Gardenes, J., Gonçalves, B., Mangioni, G., Menezes, R., Oliveira, M. (Eds.)

\footnotetext{
\begin{tabular}{l|l} 
About this book About the authors & About
\end{tabular}

\begin{tabular}{l|lll}
$\begin{array}{l}\text { About this } \\
\text { book }\end{array}$ & $\begin{array}{l}\text { About the } \\
\text { authors }\end{array}$ & $\begin{array}{l}\text { About this } \\
\text { book }\end{array}$ & $\begin{array}{l}\text { About the } \\
\text { authors }\end{array}$
\end{tabular}

This book aims to bring together researchers and practitioners from diverse disciplines-from sociology, biology, physics, and computer science-who share a passion to better understand the interdependencies within and across systems. This volume contains contributions presented at the 11th International Conference on romnlav Natinınle ICnmnlaNlatl in Evatar IInitad Kinndam 21 Marrh
}

Buy this book

\section{eBook}

\section{$117,69 €$}

price for Belgium (gross)

\section{Buy eBook}

- ISBN 978-3-030-40943-2

- Digitally watermarked, DRM-free

- Included format: PDF, EPUB

- ebooks can be used on all reading devices

- Immediate eBook download after purchase

Hardcover

$158,99 €$

» $\mathrm{FAQ}$ » Policy

\section{Services for this Book}

»Download Product Flyer

» Download High-Resolution Cover 


\section{Table of contents (33 chapters)}

Condensed Graphs: A Generic Framework

Pages 3-15 for Accelerating Subgraph Census

Computation

Martins, Miguel (et al.)

Group Cohesion Assessment in Networks

Pages 16-25 Carchiolo, v. (et al.)

Preview Buy Chapter 30,19 €

Node Classification with Bounded Error

Pages 26-38

Rates

Wijegunawardana, Pivithuru (et al.)

Preview

Buy Chapter 30,19 €

Assessment of the Effectiveness of

Pages 39-50

Random and Real-Networks Based on the

Asymptotic Entropy

Mokhlissi, Raihana (et al.)

Preview

Buy Chapter $30,19 €$

Unsupervised Strategies to Network

Pages 51-59

Topology Reconfiguration Optimization with

Limited Link Addition

Paiva, William R. (et al.)

Preview

Buy Chapter 30,19€

»Show next 20

Read this book on SpringerLink 


\section{Recommended for you}

Complex Networks $\mathrm{X}$

Cornelius, S.P. (et al.) (Eds.) (2019)

Complex Networks IX

Cornelius, S.P. (et al.) (Eds.) (2018)

Encyclopedia of Complexity and Systems Science

Editor-in-chief: Meyers, R.A. (2009)

Particles and Nuclei

Povh, B. (et al.) (2015)

The Philosophy of Quantum Physics

Friebe, C. (et al.) (2018)

Inside Interesting Integrals

Nahin, P.J. (2015)

Proceedings of NetSci-X 2020: Sixth International Winter School and Conference on Network Science

Masuda, N. (et al.) (Eds.) (2020)

Entropy and the Tao of Counting

Sharp, K. (2019)

\section{Bibliographic Information}

\section{Book Title}

Complex Networks XI

\section{Book Subtitle}

Proceedings of the 11th Conference on

Complex Networks CompleNet 2020
eBook ISBN

978-3-030-40943-2

\section{DOI}

10.1007/978-3-030-40943-2

Hardc over ISBN

\section{Number of Pages}

XVI, 404

\section{Number of Illustrations}

29 b/w illustrations, 134 illustrations in colour 
Complex Networks XI - Proceedings of the 11th Conference on Complex Networks CompleNet 2020 | Hugo Barbosa | Springer

\section{Editors}

Hugo Barbosa

Jesus Gomez-Gardenes

Bruno Gonçalves

Giuseppe Mangioni

Ronaldo Menezes

Marcos Oliveira

\section{Series Title}

» Springer Proceedings in Complexity

\section{Copyright}

2020

\section{Publisher}

Springer International Publishing

\section{Copyright Holder}

The Editor(s) (if applicable) and The

Author(s), under exclusive license to Springer Nature Switzerland AG
978-3-030-40942-5

Series ISSN

2213-8684

\section{Edition Number}

1

\section{Topics}

» Applications of Graph Theory and

Complex Networks 